\title{
Study of the Reaction Rate of Gold Nanotube Synthesis from Sacrificial Silver Nanorods through the Galvanic Replacement Method
}

\author{
Sunil Kwon, ${ }^{1}$ Hyunbae Dong, ${ }^{1,2}$ and Sang-Yup Lee ${ }^{1}$ \\ ${ }^{1}$ Department of Chemical and Biomolecular Engineering, Specialized Graduate School of Hydrogen and Fuel Cell, \\ Yonsei University, 134 Shinchon-dong, Seodaemun-gu, Seoul 120-749, Republic of Korea \\ ${ }^{2}$ Research and Development Team, Hyundai Hysco, 460-26 Sam-dong, Uiwang-Si, Gyeonggi-do 437-815, Republic of Korea
}

Correspondence should be addressed to Sang-Yup Lee, leessy@yonsei.ac.kr

Received 20 January 2010; Accepted 27 May 2010

Academic Editor: Claude Estournes

Copyright () 2010 Sunil Kwon et al. This is an open access article distributed under the Creative Commons Attribution License, which permits unrestricted use, distribution, and reproduction in any medium, provided the original work is properly cited.

\begin{abstract}
An investigation was carried out about the gold nanotube synthesis via a galvanic replacement reaction. The progress of the gold nanotube synthesis was investigated using electron microscopy and UV-Vis spectroscopy. In addition, the reaction rates of gold nanotube formation in the early stage of the reaction were studied. The chlorine ion concentration linearly increased with the gold precursor concentration but deviated from the stoichiometric amounts. This deviation was probably due to AgCl precipitates formed by the reaction of chlorine ions with dissolved silver ions. The replacement reaction was promoted with increased temperature and was nonlinearly proportional to the gold ion concentration. The outcomes of this research will enhance the current understanding of the galvanic replacement reaction.
\end{abstract}

\section{Introduction}

Metal nanotubes attract much interest due to their characteristic structure with a high aspect ratio, physical durability, and chemical activity. Compared to carbon nanotubes, metal nanotubes have enhanced reactivities toward chemicals and higher electroconductivities. These properties make metal nanotubes useful for applications in electrical devices, sensors, and catalysts among other engineering applications [1-6]. Recently, porous metal nanostructures such as metal nanotubes are also expected to be applied to not only electrical devices but also medical imaging probes because they possess surface plasmon resonance at a specific wavelength [7]. Among the many metals, gold is a promising material that is applicable for use in medical probes due to its lower toxicity than those of other metal candidates. Due to this, gold nanotubes are a promising nanomaterial with many potential applications.

For the practical production of gold nanotubes, a facile and benign synthesis method with well-understood fundamentals should be utilized. Various methods to synthesize metal nanotubes have been proposed in a number of studies. Metal nanotubes have been grown by regrowth of metal precursor on surface-modified silicon nanowires $[8,9]$, electroless deposition and subsequent etching [10], electrochemical synthesis using an inorganic anodic aluminum oxide (AAO) template [11-13], and organic tubular template-based mineralization $[14,15]$. Most of these methods are based on the template method which allows for easy control of the shape and tubular structure and can be applied for the synthesis of various hybrid materials with a core-shell structure. In addition to these methods, sacrificial templating is an interesting method in which a solid template is replaced via a galvanic reaction during the deposition of other materials. A metallic template with a lower reduction potential can be used as a sacrificial template that then controls the shape of another metal with a higher reduction potential. Solid silver nanostructures have been applied as a sacrificial template in the preparation of other porous metal and metal oxide structures. Much work has been reported by Xia et al. on shape- or morphologycontrolled nanostructures such as cubes, spheres, and other 
porous structures [16-20]. They have studied the mechanism and morphological evolution of the galvanic replacement reaction $[21,22]$. Recently, we extended the replacement reaction method traditionally used to produce solid metals so that it can be used to synthesize metal oxides [23]. Although many studies have reported on the synthesis of various porous structures, few studies have been conducted on the associated reaction rates. Detailed information of the kinetics of the replacement reaction is required for minute control of the reaction conditions.

In this paper, we studied the synthesis of gold nanotubes from sacrificial silver nanorod templates and the related reaction rates. Even though the galvanic replacement reaction takes place voluntarily due to the reduction potential difference, in practice, the reaction rate is influenced by the reaction temperature and the reactant concentrations. We investigated the effects of these factors on the replacement reaction to further understand gold nanotube formation via galvanic replacement.

\section{Experimental Section}

2.1. Chemicals. For the synthesis of silver nanorods, anhydrous ethylene glycol (99.8\%), silver nitrate $\left(\mathrm{AgNO}_{3}\right.$, 99.9\%), and polyvinyl pyrrolidone (PVP, Mw 55,000) were purchased from Aldrich. Gold(III) chloride trihydrate $\left(\mathrm{HAuCl}_{4} \cdot 3 \mathrm{H}_{2} \mathrm{O}, 99+\%\right.$, Aldrich) was used as a replaced metal ion source. All chemicals were used as received without additional purification.

2.2. Preparation of Sacrificial Silver Nanorods and Gold Nanotubes. The polyol process was used to synthesize sacrificial silver nanorods which were prepared via the growth of seed silver nanoparticles. In ethylene glycol, $\mathrm{AgNO}_{3}$ was added to generate seed silver nanocrystals. These seeds were further grown into nanorods by the capping agent. A detailed protocol for the synthesis of Ag nanorods has been presented elsewhere [23, 24].

To synthesize gold nanorods, the suspending medium of ethylene glycol was replaced with water. A $10 \mathrm{~mL}$ suspension of the synthesized $\mathrm{Ag}$ nanorods was centrifuged to collect the solid nanorods. The supernatant solution was removed, and then the nanorod aggregates were washed with acetone to remove any surface-adsorbed PVP. Removal of ethylene glycol and PVP is required to prevent the additional growth of gold [25]. The nanorods suspended in acetone were recollected by centrifugation in order to exchange the acetone suspending medium with water. The water washing process was repeated three times. The mass concentration of the silver nanorod suspension was determined by weighing the collected Ag nanorods. The gold precursor solution was prepared by dissolving $\mathrm{HAuCl}_{4}$ in deionized water to generate a solution with a concentration of $10 \mathrm{mM}$. To perform the galvanic replacement reaction, $5 \mathrm{~mL}$ of the $10 \mathrm{mM} \mathrm{HAuCl}_{4}$ solution was quickly added to $30 \mathrm{~mL}$ of the silver nanorod suspension (solid content $2.1 \mathrm{mg} / \mathrm{mL}$ ) in a glass flask under vigorous stirring at $100^{\circ} \mathrm{C}$. During the replacement reaction, the black silver nanorod solution turned gray, suggesting the formation of $\mathrm{AgCl}$. For the kinetics study, the reaction was performed at different temperatures of 25,40 , and $60^{\circ} \mathrm{C}$. The reaction was allowed to proceed for $20 \mathrm{~min}$, during which, samples were taken to monitor the concentration of the remaining gold ions.

2.3. Characterization. The synthesized silver nanorods and gold nanotubes were investigated using field emission scanning electron microscopy (FE-SEM, Hitachi S-4200, Nissei Sangyo Co., $30 \mathrm{kV}$ ) and energy-filtering transmission electron microscopy (EF-TEM, LIBRA 120, Zeiss Co., $120 \mathrm{kV}$ ). The chemical composition of the nanoparticles was confirmed by energy dispersive X-ray analysis (EDX). UVVis absorption spectra were obtained (UV-Vis, 1601 PC, Shimadzu Co.) before and after the replacement reaction. The concentration of the remaining gold ions was determined by measuring the UV-Vis absorbance intensity at a wavelength of $310 \mathrm{~nm}$ after preparing a standard curve [26]. The absorbance intensity may contain the effect of interband absorption which cannot be eliminated in the measurement.

\section{Results and Discussion}

3.1. Synthesis of Gold Nanotubes. The sacrificial silver nanorod templates were prepared via the polyol process in which silver nanoparticles were used as seeds for nanorod growth. To direct the c-axial growth of the seed nanoparticles, PVP acted as a capping molecule directing the silver growth [24]. The silver nanoparticles used as seeds are shown in Figure 1(a). The seed nanoparticles had an average particle size of $11.9 \pm 9.1 \mathrm{~nm}$. The prepared sacrificial silver nanorods are shown in Figure 1(b). The Ag nanorods had an average diameter of $100.3 \pm 14.8 \mathrm{~nm}$ and were several micrometers in length. As seen in the TEM image, some spherical silver particles were also observed whose growth may not have been properly adjusted by the capping molecules. From the magnified image of the silver nanorods, they were seen to have smooth surfaces with a round end (Figure 1(c)). The axial growth of the silver nanorods was characterized by the XRD study in which the (111) peak shows the strongest intensity (data not shown). The growth of the silver nanorods was also characterized by evaluating the changes in the UV-Vis spectra. Silver nanoparticles are known to exhibit surface plasmon resonance in the UV-Vis region, such that comparison between nanoparticles and nanorods is possible [27]. The spectrum of the silver seeds has a characteristic peak at $425 \mathrm{~nm}$. This type of UV-Vis spectrum is commonly observed with many metal nanoparticles and is influenced by their size. After formation of the nanorods, the characteristic $\mathrm{Ag}$ seed UV-Vis spectrum peak disappeared, and broad nanorod peak showed up. Due to the increase of anisotropy of the suspending particles as well as the size increase of the dispersed rod-like particle, the UV-Vis spectrum was broadened, and its absorbance was diminished.

From the synthesized sacrificial Ag nanorods, the $\mathrm{Au}$ nanotubes were prepared. Preparation of the Au nanotubes was performed via the galvanic replacement reaction using a $10 \mathrm{mM} \mathrm{HAuCl}_{4}$ solution. The reaction is a voluntarily 


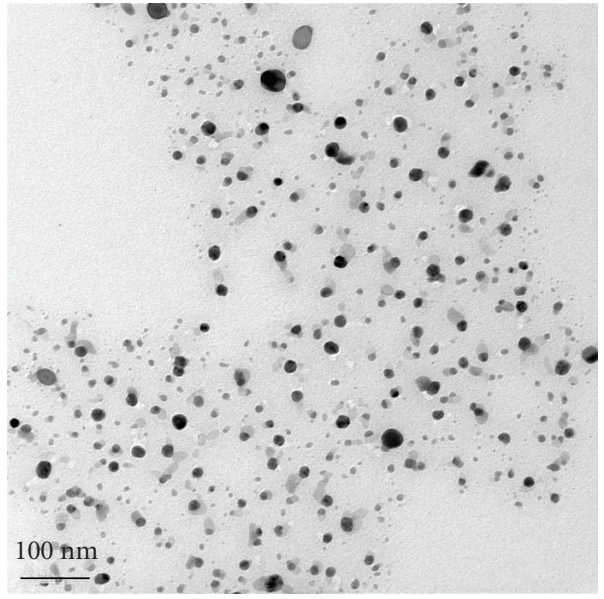

(a)

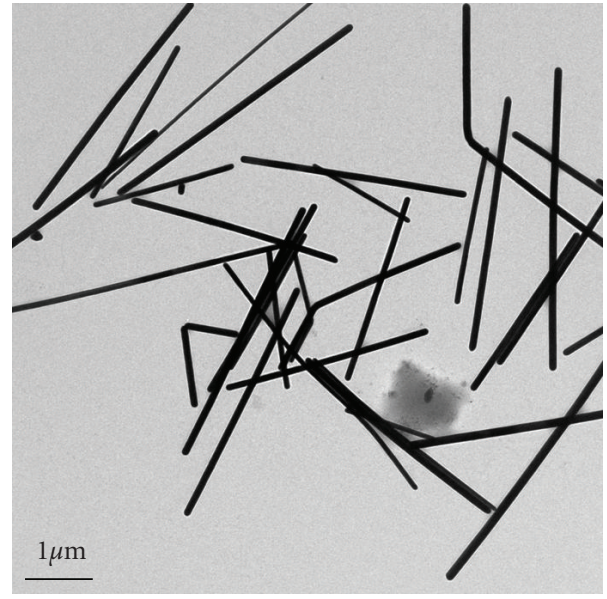

(b)

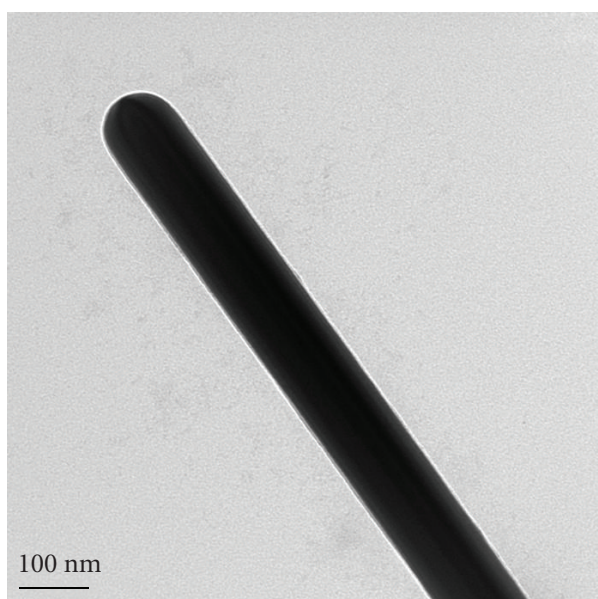

(c)

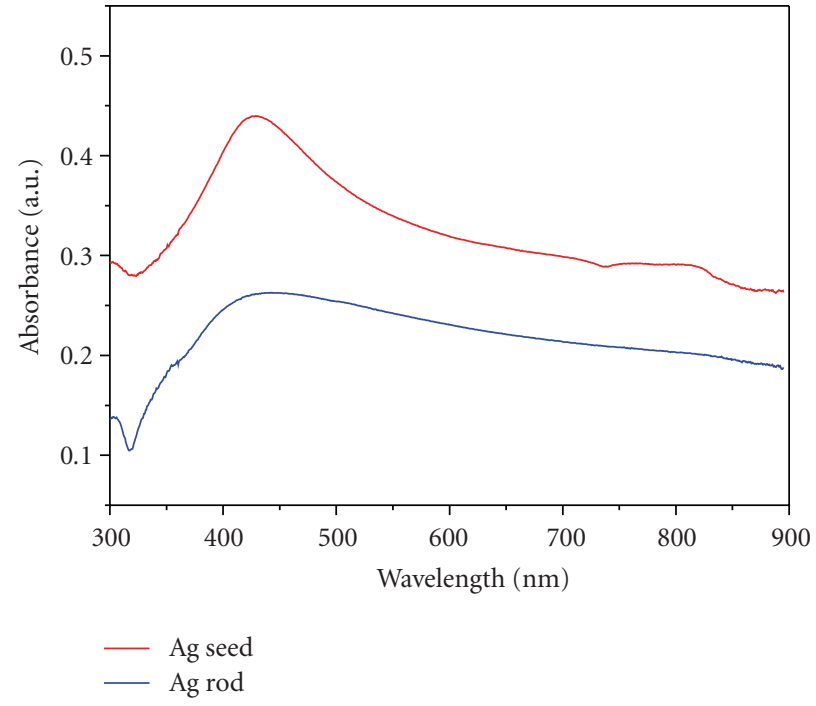

(d)

FIGURE 1: Characteristics of the sacrificial Ag nanorods. (a) TEM image of the seed silver nanoparticles (scale bar: $100 \mathrm{~nm}$ ), (b) TEM image of the synthesized sacrificial Ag nanorods (scale bar: $1 \mu \mathrm{m}$ ), (c) magnified image of the sacrificial Ag nanorods (scale bar: $100 \mathrm{~nm}$ ), and (d) UV-Vis spectroscopy of the Ag seeds and nanorods.

reaction in which the substitution of the $\operatorname{Ag}(s)$ core with $\mathrm{Au}(\mathrm{s})$ is driven by the difference in their standard reduction potentials. The standard reduction potentials of $\mathrm{Ag}$ and $\mathrm{Au}$ ions at $25^{\circ} \mathrm{C}$ are shown below [28]:

$$
\begin{gathered}
\mathrm{Ag}^{+}(\mathrm{aq})+\mathrm{e}^{-} \longrightarrow \mathrm{Ag}(\mathrm{s}), \quad \mathrm{E}^{0}=+0.80 \mathrm{~V}, \\
\mathrm{AuCl}_{4}{ }^{-}(\mathrm{aq}) \longrightarrow \mathrm{Au}(\mathrm{s})+4 \mathrm{Cl}^{-}, \quad \mathrm{E}^{0}=+1.00 \mathrm{~V} .
\end{gathered}
$$

The higher standard reduction potential of the gold ion enables the replacement reaction to proceed spontaneously such that the following chemical reaction is possible:

$$
3 \mathrm{Ag}(\mathrm{s})+\mathrm{AuCl}_{4}^{-}(\mathrm{aq}) \longrightarrow 3 \mathrm{Ag}^{+}(\mathrm{aq})+\mathrm{Au}(\mathrm{s})+4 \mathrm{Cl}^{-}(\mathrm{aq}) \text {. }
$$

The solid silver rod is the source of electrons similar to other galvanic replacement reactions, and a gold nanotube template is formed simultaneously.
Images of the synthesized porous $\mathrm{Au}$ nanotubes are shown in Figures 2(a) and 2(b). As seen in the TEM images, a cavity in the $\mathrm{Au}$ nanorods is present at lower intensity. The SEM image shows a continuous gold layer, and crystallinity of the gold shell was not observed. The magnified image, Figure 2(c), shows the detailed structure of the porous gold nanotube whose surfaces were roughened after the replacement reaction. To confirm the replacement of gold with silver, energy dispersive X-ray analysis (EDXA) was performed. Strong gold peaks at around $10 \mathrm{keV}$ as well as silver peaks are observed in Figure 2(d). The silver peaks come from the remnant of Ag. The copper peaks result from the TEM grid used in the measurements.

The replacement reaction using the gold precursor was promoted by increasing the gold precursor concentration. In Figure 3, the UV-Vis spectra after the replacement reaction are shown at different gold precursor concentrations. 


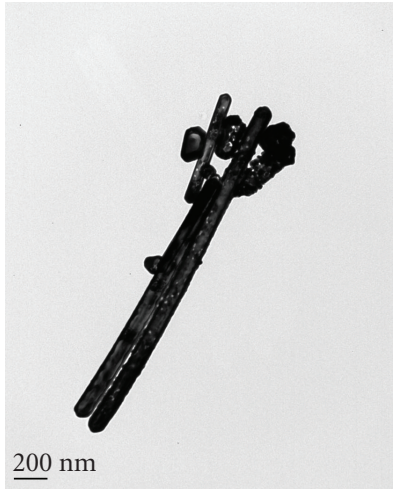

(a)

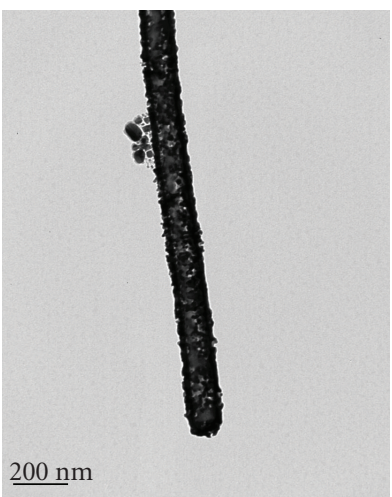

(c)

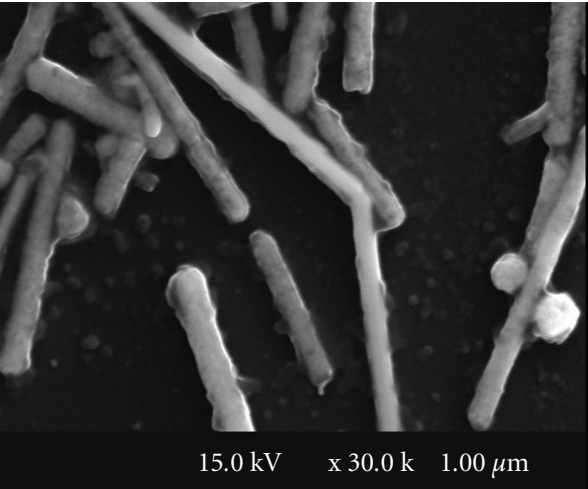

(b)

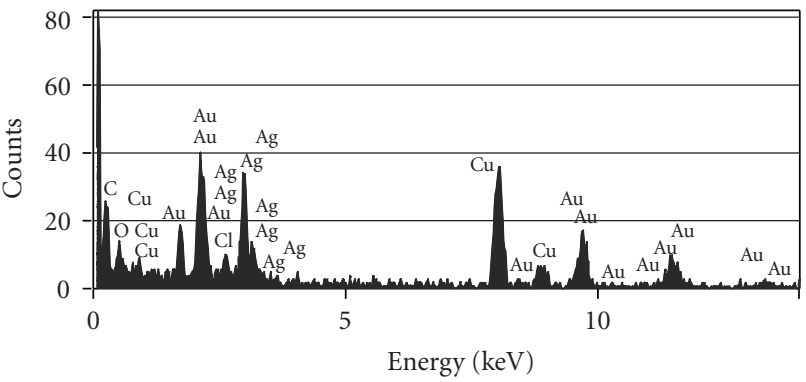

(d)

FIgURE 2: Characteristics of synthesized gold nanotubes. (a, b) TEM and SEM images of the prepared gold nanotubes (scale bar: 200 nm), (c) magnified image of the gold nanotubes (scale bar: $200 \mathrm{~nm}$ ), and (d) EDXA peaks of the porous gold nanotubes.

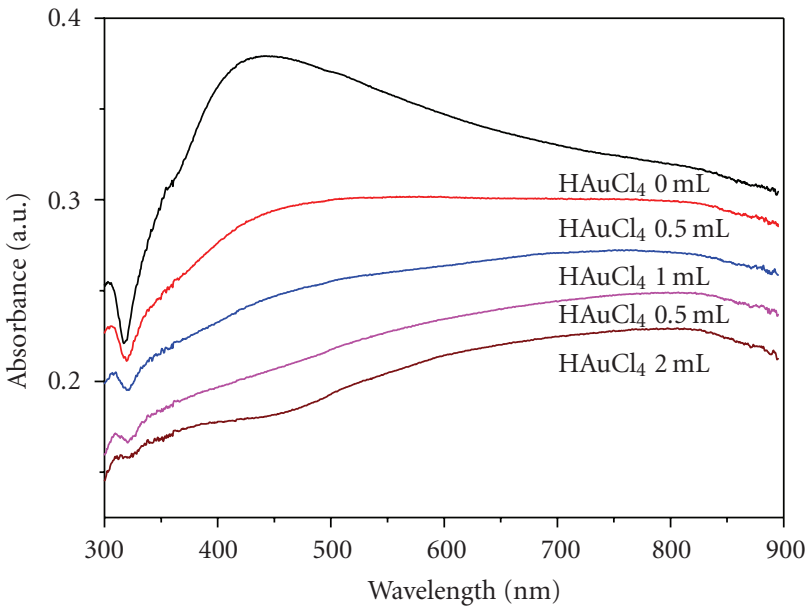

FIGURE 3: UV-Vis spectra of the gold nanorods prepared at different gold precursor concentrations.

The UV-Vis spectra were taken after 20 minutes of the replacement reaction at $100^{\circ} \mathrm{C}$ with different gold precursor concentrations. With increasing gold precursor concentration, the replacement of silver nanorods and gold layer

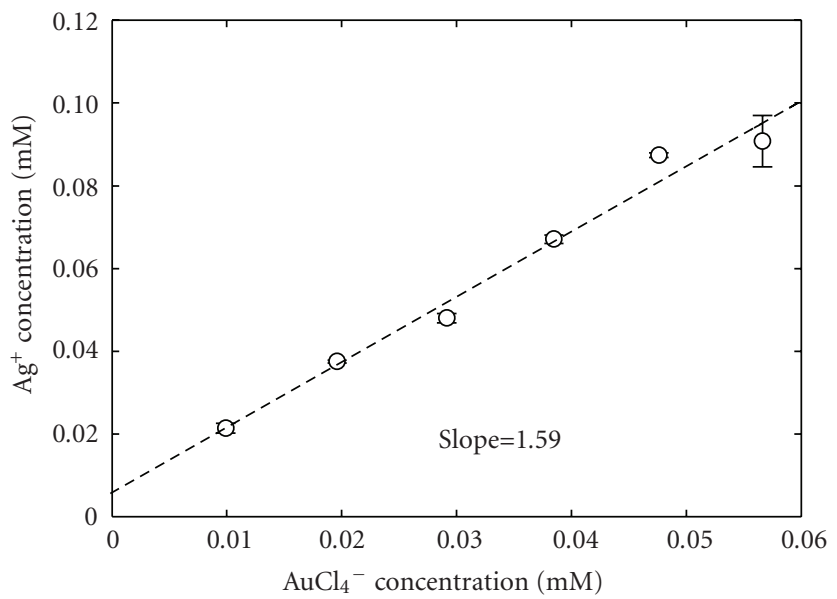

Figure 4: Concentrations of silver ions dissolved from the solid silver nanorods along with the gold precursor concentration.

formation were promoted such that peak broadening and a red shift of the spectrum were observed.

\subsection{Effects of Reaction Conditions on the Gold Nanotube Formation. To investigate the replacement reaction in detail,}




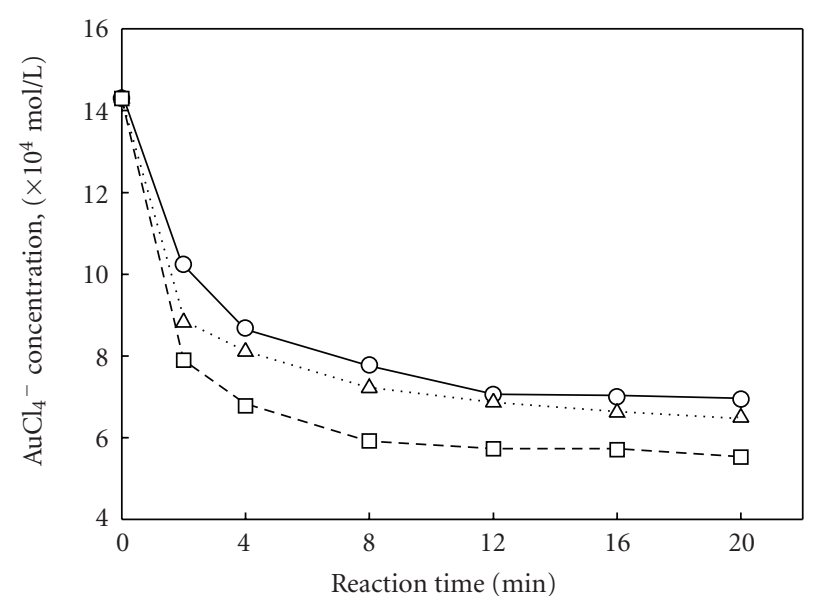

$$
\begin{aligned}
& -\mathrm{O}-25^{\circ} \mathrm{C} \\
& \cdot \Delta \quad 40^{\circ} \mathrm{C} \\
& -\square-60^{\circ} \mathrm{C}
\end{aligned}
$$

(a)
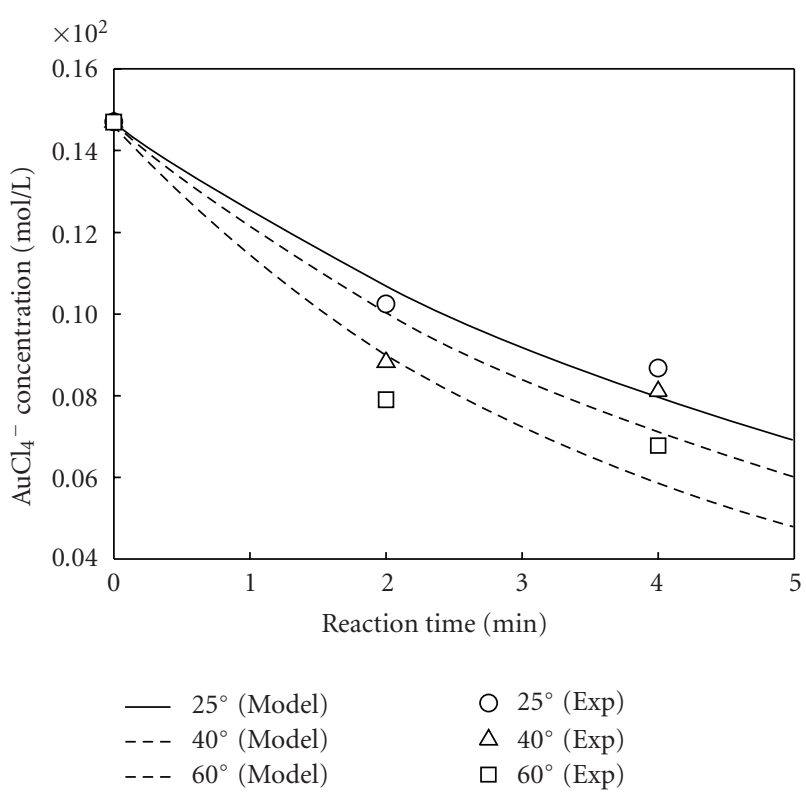

(b)

Figure 5: (a) Gold ion concentration as a function of time at different reaction temperatures (lines are guide for eye), (b) the fitted line of the reaction rate equation determined from the data points.

the concentration of dissolved $\mathrm{Ag}$ ions after the galvanic replacement reaction was monitored. In addition, the degree of galvanic replacement was determined while varying the gold precursor ion concentration. The silver ion concentration in the aqueous solution was monitored after completion of replacement with gold. The concentration of dissolved silver ions increased linearly with the gold precursor concentration, as seen in Figure 4. It is obvious that the replacement reaction is directly proportional to the precursor concentration. The slope of the regression curve was 1.59 , which is much lower than the stoichiometric number of 3 in the replacement reaction in (3). Deviation from stoichiometry may be due to the hydrolysis of $\mathrm{AuCl}_{4}{ }^{-}$ ions in the aqueous solution, which is influenced by $\mathrm{pH}$. When the gold precursor solution ( $\mathrm{pH}$ 2.92) was dissolved in the silver nanorod solution, the $\mathrm{pH}$ of the nanorod solution was 5.28. At this $\mathrm{pH}$, the expected hydrolyzed $\mathrm{AuCl}_{4}{ }^{-}$ion structure is $\left[\mathrm{AuCl}_{2.1}(\mathrm{OH})_{1.9}\right]^{-}$releasing $\sim 1.9 \mathrm{Cl}^{-}$ions [29]. The released $\mathrm{Cl}^{-}$ions repress the reduction of gold chloride ions such that the experimental results deviate from the stoichiometry of the replacement reaction. This result is in agreement with the trend of reduction potential decrease with $\mathrm{pH}$ increase [29]. Another potential explanation for the deviation from stoichiometry is the precipitation of $\mathrm{AgCl}$, which consumes dissolved $\mathrm{Ag}^{+}$ions via reaction with $\mathrm{Cl}^{-}$ ions. After the galvanic replacement, white water-insoluble precipitates of $\mathrm{AgCl}$ were observed (data not shown) when the reactant was cooled to room temperature. Unfortunately, we were not able to separate the white precipitates from the gold and silver nanorod particles to quantify their amounts.
To investigate the reaction rate, the progress of the replacement reaction was monitored by measuring the gold precursor concentration. In Figure 5(a), the concentrations of the $\mathrm{AuCl}_{4}{ }^{-}$ions are plotted as a function of time at various reaction temperatures. As seen in the results, the replacement of solid silver nanorods with gold ions is promoted at increased temperature. The concentration profile indicates that most of the reaction is completed during the early stage of reaction (in the first ten minutes).

The reaction rate of the galvanic replacement is influenced by the concentrations of both the solid $\mathrm{Ag}$ nanorods and the $\mathrm{AuCl}_{4}{ }^{-}$ions. However, the reaction rate of $\mathrm{AuCl}_{4}{ }^{-}$ ions, $r_{\mathrm{AuCl}_{4}}$, is simply expressed as (4) under the assumption of a constant silver nanorod concentration. The simplified rate equation is only a function of the concentration of $\mathrm{AuCl}_{4}{ }^{-}$with the reaction rate constant $k$ and the order of the reaction $\beta$ :

$$
r_{\mathrm{AuCl}_{4}{ }^{-}}=-k C_{\mathrm{AuCl}_{4}{ }^{-}}^{\beta} .
$$

The above rate equation is valid when the concentration of silver nanorods is much higher than that of $\mathrm{AuCl}_{4}{ }^{-}$, as well as in the early stage of the reaction. In practice, this assumption is advantageous because determination of the molar concentration of silver nanorods is not practical and most of the replacement reaction is completed in the early stage. In the present experiments, the silver nanorod concentration was set at $2.1 \mathrm{mg} / \mathrm{mL}$, which is around four times higher than that of $\mathrm{HAuCl}_{4}(1.43 \mathrm{mM}$ in the mixture which corresponds to $\sim 0.56 \mathrm{mg} / \mathrm{mL}$ ), and the data in the early stage of the reaction was used for the analysis. Since the replacement reaction is taking place in a batch type reactor, 
TABLE 1: Reaction rate constant, $k$, at various temperatures.

\begin{tabular}{lccc}
\hline Temperature $\left[{ }^{\circ} \mathrm{C}\right]$ & 25 & 40 & 60 \\
\hline$k\left[(\mathrm{~L} / \mathrm{mol})^{0.24} \cdot \mathrm{min}^{-1}\right]$ & 0.780 & 0.943 & 1.225 \\
\hline
\end{tabular}

upon integration, (4) can be written as (5) using the initial condition of $C_{\mathrm{Aucl}_{4}{ }^{-}}=C_{\left(\mathrm{Aucl}_{4}{ }^{-}\right)_{0}}$ :

$$
t=\frac{1}{k} \frac{C_{\left(\mathrm{Aucl}_{4}{ }^{-}\right)_{0}}^{(1-\beta)}-C_{\mathrm{Aucl}_{4}{ }^{-}}^{(1-\beta)}}{1-\beta}
$$

The $k$ and $\beta$ parameters were determined by nonlinear regression using Polymath with the data sets in the early stage of the reaction. After the data fitting, the order of the reaction, $\beta$, was determined to be 1.24 , while the reaction rate constant $k$ varied according to the reaction temperature. The reaction rate constant $k$ results are summarized in Table 1. As expected, $k$ increases with temperature. These values are applicable for the early stage of the replacement reaction of gold ions with solid silver, which explains the promoted replacement reaction at increased temperature

\section{Conclusions}

The galvanic replacement method was applied for the synthesis of gold nanotubes from sacrificial silver nanorods. The progress of the replacement reaction was monitored by obtaining UV-Vis spectra and by measuring the silver and gold ion concentrations. The replacement reaction was promoted by increased gold precursor concentration as well as by increased reaction temperature. The dissolved silver concentration increased linearly with the gold ion concentration. The replacement reaction was promoted with increased temperature, which enhances the reaction rate constant. The reaction rate depends on the gold precursor concentration nonlinearly. This study determined kinetic information about gold replacement with sacrificial silver nanorod templates through the galvanic replacement reaction.

\section{Acknowledgment}

This work was supported by Korea Science and Engineering Foundation (KOSEF M10755020001-08N5502-00110). S. Kwon and H. Dong contributed equally to the paper.

\section{References}

[1] W. Y. Kim, Y. C. Choi, and K. S. Kim, "Understanding structures and electronic/spintronic properties of single molecules, nanowires, nanotubes, and nanoribbons towards the design of nanodevices," Journal of Materials Chemistry, vol. 18, no. 38, pp. 4510-4521, 2008.

[2] M. Lee, S. Hong, and D. Kim, "Chemical-free synthesis of electrically connected gold nanotubes/ nanoparticles from solution-infiltrated anodized aluminum oxide template," Applied Physics Letters, vol. 89, no. 4, Article ID 043120, 3 pages, 2006.
[3] S. Yu, U. Welp, L. Z. Hua, A. Rydh, W. K. Kwok, and H. H. Wang, "Fabrication of palladium nanotubes and their application in hydrogen sensing," Chemistry of Materials, vol. 17, no. 13, pp. 3445-3450, 2005.

[4] R. J. Chen, H. C. Choi, S. Bangsaruntip et al., "An investigation of the mechanisms of electronic sensing of protein adsorption on carbon nanotube devices," Journal of the American Chemical Society, vol. 126, no. 5, pp. 1563-1568, 2004.

[5] H. Chen, S. Chen, X. Quan, H. Yu, H. Zhao, and Y. Zhang, "Fabrication of $\mathrm{TiO}_{2}-\mathrm{Pt}$ coaxial nanotube array Schottky structures for enhanced photocatalytic degradation of phenol in aqueous solution," Journal of Physical Chemistry C, vol. 112, no. 25, pp. 9285-9290, 2008.

[6] T.-Y. Shin, S.-H. Yoo, and S. Park, "Gold nanotubes with a nanoporous wall: their ultrathin platinum coating and superior electrocatalytic activity toward methanol oxidation," Chemistry of Materials, vol. 20, no. 17, pp. 5682-5686, 2008.

[7] J. Chen, F. Saeki, B. J. Wiley et al., "Gold nanocages: bioconjugation and their potential use as optical imaging contrast agents," Nano Letters, vol. 5, no. 3, pp. 473-477, 2005.

[8] Y. Qu, R. Porter, F. Shan, J. D. Carter, and T. Guo, "Synthesis of tubular gold and silver nanoshells using silica nanowire core templates," Langmuir, vol. 22, no. 14, pp. 6367-6374, 2006.

[9] Y. Qu, J. D. Carter, A. Sutherland, and T. Guo, "Surface modification of gold nanotubules via microwave radiation, sonication and chemical etching," Chemical Physics Letters, vol. 432, no. 1-3, pp. 195-199, 2006.

[10] S. L. Cheng and W. C. Hsiao, "Electroless synthesis of pure nickel metal nanotubes using silicon oxide nanowires as removable templates," Electrochemical and Solid-State Letters, vol. 10, no. 12, pp. D142-D145, 2007.

[11] W. Lee, R. Scholz, K. Nielsch, and U. Gösele, "A templatebased electrochemical method for the synthesis of multisegmented metallic nanotubes," Angewandte Chemie International Edition, vol. 44, no. 37, pp. 6050-6054, 2005.

[12] D. Yang, G. Meng, S. Zhang et al., "Electrochemical synthesis of metal and semimetal nanotube-nanowire heterojunctions and their electronic transport properties," Chemical Communications, no. 17, pp. 1733-1735, 2007.

[13] T. Sehayek, M. Lahav, R. Popovitz-Biro, A. Vaskevich, and I. Rubinstein, "Template synthesis of nanotubes by roomtemperature coalescence of metal nanoparticles," Chemistry of Materials, vol. 17, no. 14, pp. 3743-3748, 2005.

[14] S.-Y. Lee, E. Royston, J. N. Culver, and M. T. Harris, "Improved metal cluster deposition on a genetically engineered tobacco mosaic virus template," Nanotechnology, vol. 16, no. 7, pp. S435-S441, 2005.

[15] R. Djalali, Y.-F. Chen, and H. Matsui, "Au nanowire fabrication from sequenced histidine-rich peptide," Journal of the American Chemical Society, vol. 124, no. 46, pp. 13660-13661, 2002.

[16] Y. Sun and Y. Xia, "Multiple-walled nanotubes made of metals," Advanced Materials, vol. 16, no. 3, pp. 264-268, 2004.

[17] J. Chen, J. M. McLellan, A. Siekkinen, Y. Xiong, Z.-Y. Li, and Y. Xia, "Facile synthesis of gold-silver nanocages with controllable pores on the surface," Journal of the American Chemical Society, vol. 128, no. 46, pp. 14776-14777, 2006.

[18] Y. Sun, B. Wiley, Z.-Y. Li, and Y. Xia, "Synthesis and optical properties of nanorattles and multiple-walled nanoshells/nanotubes made of metal alloys," Journal of the American Chemical Society, vol. 126, no. 30, pp. 9399-9406, 2004.

[19] Y. Sun, B. T. Mayers, and Y. Xia, “Template-engaged replacement reaction: a one-step approach to the large-scale synthesis 
of metal nanostructures with hollow interiors," Nano Letters, vol. 2, no. 5, pp. 481-485, 2002.

[20] Y. Sun and Y. Xia, "Increased sensitivity of surface plasmon resonance of gold nanoshells compared to that of gold solid colloids in response to environmental changes," Analytical Chemistry, vol. 74, no. 20, pp. 5297-5305, 2002.

[21] Y. Sun and Y. Xia, "Mechanistic study on the replacement reaction between silver nanostructures and chloroauric acid in aqueous medium," Journal of the American Chemical Society, vol. 126, no. 12, pp. 3892-3901, 2004.

[22] M. H. Kim, X. Lu, B. Wiley, E. P. Lee, and Y. Xia, "Morphological evolution of single-crystal Ag nanospheres during the galvanic replacement reaction with $\mathrm{HAuCl}_{4}$," Journal of Physical Chemistry C, vol. 112, no. 21, pp. 7872-7876, 2008.

[23] H. Dong, E. K. Koh, and S.-Y. Lee, "Synthesis of Ag$\mathrm{Mn}_{3} \mathrm{O}_{4}$ core-shell nanorods and $\mathrm{Mn}_{3} \mathrm{O}_{4}$ nanotubes from sacrificial Ag nanorod templates," Journal of Nanoscience and Nanotechnology, vol. 9, no. 11, pp. 6511-6517, 2009.

[24] Y. Cheng, Y. Wang, C. Jia, and F. Bao, "MnS hierarchical hollow spheres with novel shell structure," Journal of Physical Chemistry B, vol. 110, no. 48, pp. 24399-24402, 2006.

[25] P.-Y. Silvert, "Preparation of colloidal silver dispersions by the polyol process. Part 1-synthesis and characterization," Journal of Materials Chemistry, vol. 6, no. 4, pp. 573-577, 1996.

[26] J.-E. Park, M. Atobe, and T. Fuchigami, "Sonochemical synthesis of conducting polymer-metal nanoparticles nanocomposite," Electrochimica Acta, vol. 51, no. 5, pp. 849-854, 2005.

[27] M. Kerker, "The optics of colloidal silver: something old and something new," Journal of Colloid And Interface Science, vol. 105, no. 2, pp. 297-314, 1985.

[28] M. Ware, "An investigation of platinum and palladium printing," Journal of Photographic Science, vol. 34, pp. 13-25, 1986.

[29] S. Wang, K. Qian, X. Bi, and W. Huang, "Influence of speciation of aqueous $\mathrm{HAuCl}_{4}$ on the synthesis, structure, and property of Au colloids," Journal of Physical Chemistry C, vol. 113, no. 16, pp. 6505-6510, 2009. 

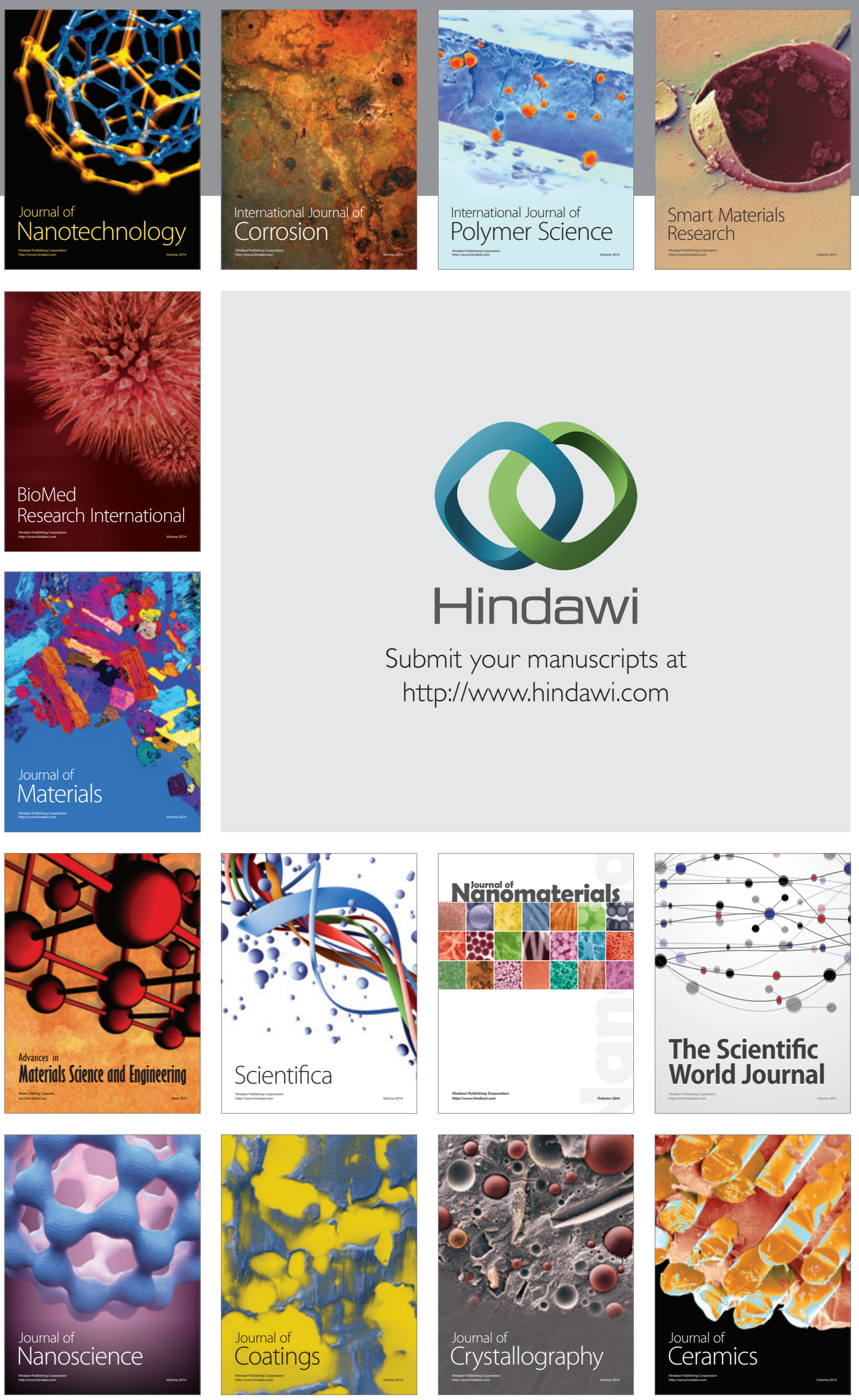

The Scientific World Journal

Submit your manuscripts at

http://www.hindawi.com

\section{World Journal}

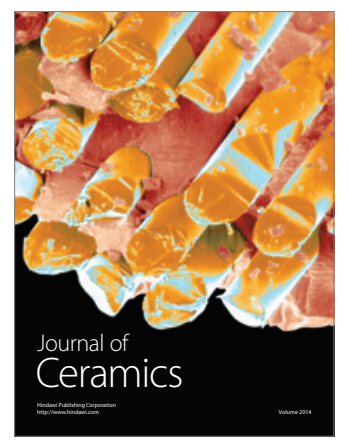

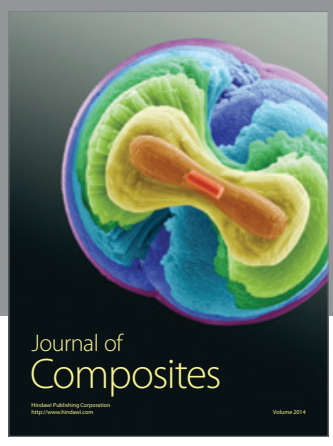
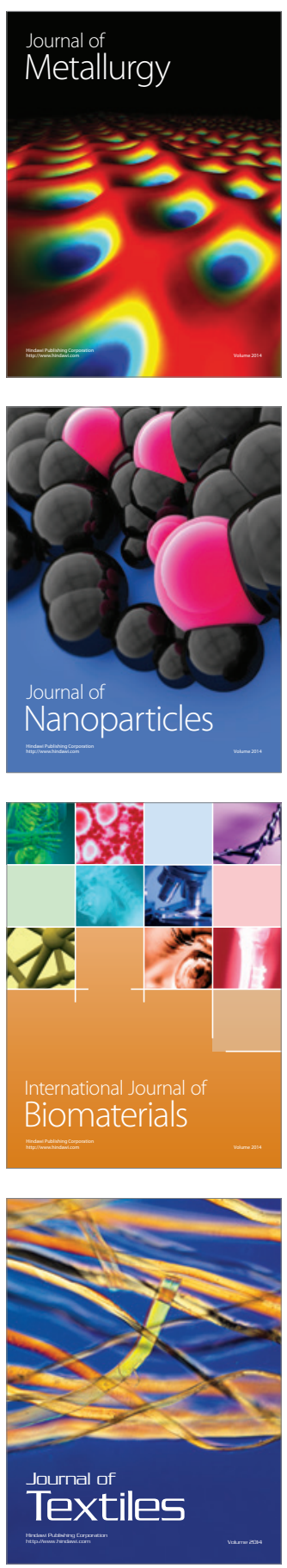